\title{
Gesund heits-
}

und Sozialpolitik $\begin{gathered}\text { Zeitschriff tiut das gesante } \\ \text { cesundheitswesen }\end{gathered}$

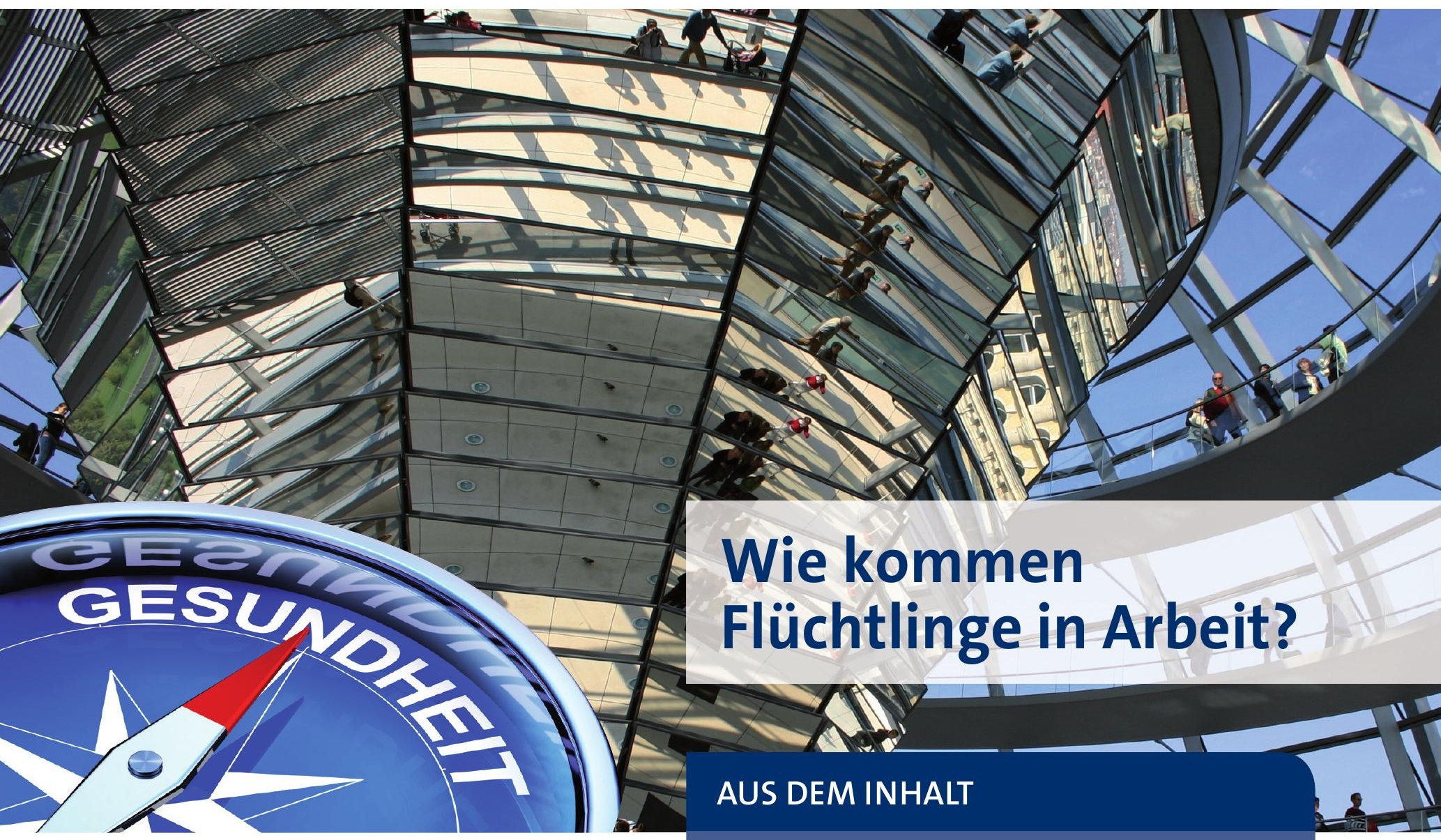

Ulrich Walwei

Fluchtmigration und Arbeitsmarkt: Kann die Integration gelingen?

Björn Broge, Klaus Focke, Benjamin Finger Entlassmanagement: Paradigmenwechsel durch

\section{6/2016} Kooperation und Überwindung von Sektorengrenzen Reinhild Bücheler et al

Webbasierte Informationsplattform zur rationalen und wirtschaftlichen Pharmakotherapie -

70. Jahrgang

ISSN 1611-5821

Ein Beitrag zu mehr Verordnungssicherheit für Vertragsärzte?

Thomas Czihal, Dominik von Stillfried

Konsequenzen der Flexibilisierung des Zulassungsrechts auf die Produktivität in der vertragsärztlichen Versorgung

\section{Nomos}

Angelika Seger, Wolfgang Seger

Chancen, Risiken und Grenzen einer Qualitätsorientierten Vergütung im internationalen Kontext -57 


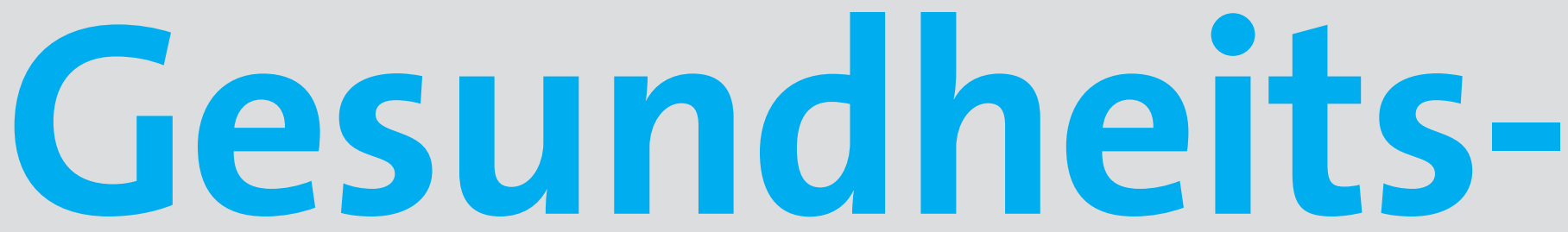
und Sozialpolitik

BEIRAT: Prof. Dr. med. Reinhard Busse, Technische Universität Berlin, Prof. Josef Hecken, Unparteiischer Vorsitzender des Gemeinsamen Bundesausschusses, Berlin, Dr. Christopher Hermann, Vorsitzender des Vorstandes der AOK Baden-Württemberg, Stuttgart, Ralf Heyder, Generalsekretär des Verbands der Universitätsklinika Deutschlands, Berlin, Prof. Dr. med. David Klemperer, Ostbayerische Technische Hochschule Regensburg, Dr. Volker Leienbach, PKV-Verbandsdirektor, Köln, Prof. Dr. Karl Lauterbach, Mitglied des Deutschen Bundestages, Berlin, Prof. Dr. Heinz Rothgang, Universität Bremen, Prof. Dr. med. Matthias Schrappe, Bonn, Prof. Dr. Heinz-Dietrich Steinmeyer, Universität Münster, Dr. Christoph Straub, Vorstandsvorsitzender der BARMER GEK, Berlin, Prof. Dr. Leonie Sundmacher, Ludwig-Maximilians-Universität München, Prof. Dr. Sascha Wolf, Hochschule Pforzheim

HERAUSGEBER: Prof. Volker E. Amelung, Franz Knieps, Karl-Heinz Schönbach

REDAKTEUR: Erwin Dehlinger (ViSdP)

\section{EDITORIAL}

\section{TRENDS \& FACTS}

\section{THEMA}

Fluchtmigration und Arbeitsmarkt:

Kann die Integration gelingen?

Ulrich Walwei

Entlassmanagement: Paradigmenwechsel durch Kooperation und Überwindung von Sektorengrenzen

Björn Broge, Klaus Focke, Benjamin Finger

Webbasierte Informationsplattform zur rationalen und wirtschaftlichen Pharmakotherapie - Ein Beitrag zu mehr Verordnungssicherheit für Vertragsärzte? Reinhild Bücheler et al

Konsequenzen der Flexibilisierung des Zulassungsrechts auf die Produktivität in der vertragsärztlichen Versorgung

Thomas Czihal, Dominik von Stillfried

Chancen, Risiken und Grenzen einer Qualitätsorientierten Vergütung im internationalen Kontext - Ein Modell für Deutschland?

Angelika Seger, Wolfgang Seger

Sicherstellung pflegerischer

Langzeitversorgung - Strategien zur

Steigerung ehrenamtlichen Engagements

Christine Niens et al

Unfallversicherungsträger und

Gesundheitsförderung an Hochschulen im

Fokus des Präventionsgesetzes - Potenziale und Perspektiven für Studierende

Sandra Tschupke, Thomas Hartmann

\section{ZUR DISKUSSION}

Evidenz - Glaube - politische Adelung: Positionen zur so genannten Komplementärmedizin Norbert Schmacke

Die kieferorthopädische Versorgung im SGB V-unzweckmäßig, intransparent und paternalistisch

Alexander Spassov, Hartmut Bettin, Bernard Braun

Zurück zur paritätischen Finanzierung der Gesetzlichen Krankenversicherung?

Wolfram F. Richter

\section{REZENSIONEN}

IMPRESSUM
Fluchtmigration und Arbeitsmarkt: Kann die Integration gelingen?

Seite 6 Schätzungen zufolge sind 2015 knapp 900.000 Menschen nach Deutschland geflüchtet. Für 2016 wird davon ausgegangen, dass noch einmal rund 300.000 dazu kommen könnten. Der Beitrag gibt erste Antworten auf die Frage, wie der Arbeitsmarkt die Fluchtmigration verkraften wird und ob sich geflüchtete Menschen mit nachhaltigem Erfolg in den Arbeitsmarkt integrieren können. Hierzu werden Forschungsergebnisse aus dem Institut für Arbeitsmarkt- und Berufsforschung präsentiert.

6 Entlassmanagement: Paradigmenwechsel durch Kooperation und Überwindung von Sektorengrenzen

Seite 17

Mit dem „Rahmenvertrag Entlassmanagement“ hat das Erweiterte Bundesschiedsamt formale Grundlagen festgelegt, damit der Versorgungsbedarf von Patienten im Anschluss an eine Krankenhausbehandlung zukünftig besser geplant und umgesetzt wird. Dazu muss jedoch das vorhandene Wissen über sinnvolle Inhalte eines Entlassmanagements zunächst so strukturiert werden, dass es in den formalen Rahmen passt und eine transparente Struktur für alle Beteiligten zur Verfügung steht. Die hierbei zu berücksichtigenden Handlungsfelder werden im Artikel dargestellt.

Webbasierte Informationsplattform zur rationalen und wirtschaftlichen PharmakotherapieEin Beitrag zu mehr Verordnungssicherheit für Vertragsärzte?

Seite 22

Die Ausstellung einer medizinisch adäquaten, haftungsrechtlich und ökonomisch unangreifbaren Arzneimittelverordnung ist keine triviale Aufgabe. Die Angst vor Arzneimittelregressen kann dazu führen, dass medizinisch notwendige Arzneimittel Patienten vorenthalten werden. In einem bislang einmaligen Kooperationsprojekt haben die Vertragsparteien in Baden-Württemberg begonnen, Therapieleitfäden/-empfehlungen zu konsentieren und sie auf einer frei zugänglichen Internetplattform bereitzustellen. Der Beitrag beschreibt das Projekt und erste Reaktionen.

Konsequenzen der Flexibilisierung des Zulassungsrechts auf die Produktivität in der vertragsärztlichen Versorgung

Seite 27 Das Vertragsarztrechtsänderungsgesetz hat das Zulassungsrecht maßgeblich reformiert. Damit sollte die vertragsärztliche Berufsausübung „effizienter und damit wettbewerbsfähiger“ gestaltet werden. Konnten diese Ziele erreicht werden? Zehn Jahre nach Inkrafttreten der Reform können die Auswirkungen analysiert werden. Insbesondere die Produktivität bzw. die Arbeitszeit je Arzt hat sich erheblich verändert. Die Planungsinstrumente in der gesetzlichen Krankenversicherung berücksichtigen die Reformauswirkungen jedoch nicht ausreichend.

Chancen, Risiken und Grenzen einer Qualitätsorientierten Vergütung im internationalen Kontext - Ein Modell für Deutschland?

Seite 32

Der Beitrag stellt die Anforderungen an eine qualitätsorientierte Vergütung im Gesundheitswesen im internationalen Kontext dar. Empfohlen wird die Einführung der qualitätsorientierten Vergütung in Deutschland in Form eines lernenden Systems. Rechtlich ist die Umsetzung bereits in Modellprojekten möglich, bei einer flächenendeckenden und wirksamen Implementierung bedarf es innovativer gesetzlicher und insbesondere auch datenschutzrechtlicher Regelungen.

Sicherstellung pflegerischer Langzeitversorgung - Strategien zur Steigerung ehrenamtlichen Engagements

Seite 42 Die Sicherstellung einer bedarfsgerechten pflegerischen Langzeitversorgung zählt zu den zentralen Herausforderungen. Regionale Versorgungskonzepte und die verstärkte Einbindung Ehrenamtlicher werden als mögliche Lösung angeführt. Auf Basis eines Konzepts der Bertelsmann Stiftung betrachtet der Beitrag, inwieweit regionale Konzepte erfolgsversprechend sind, wie viele zusätzliche Ehrenamtsstunden benötigt würden und wie die Aktivierung Ehrenamtlicher gelingen kann. 


\section{Die fließende Zeit und das flüchtige Recht}

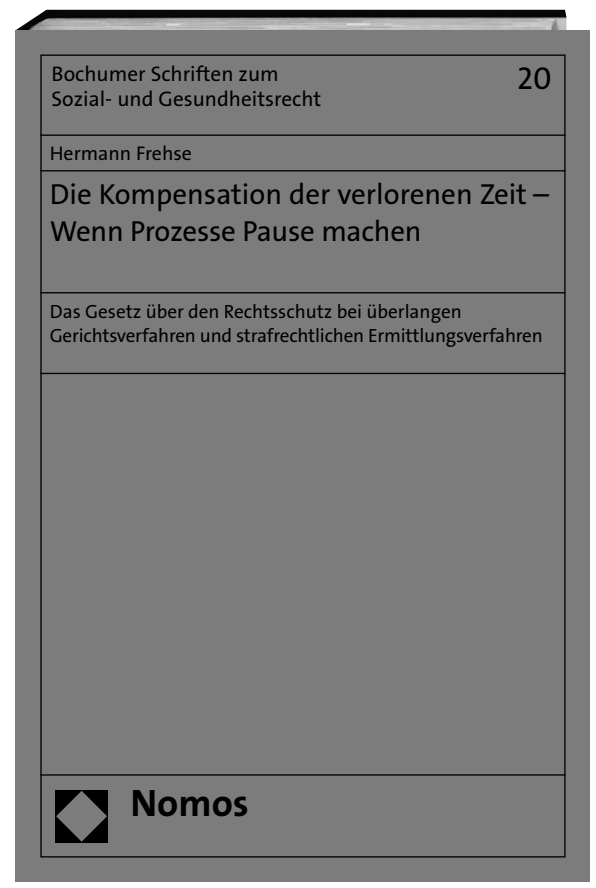

Nicht zeitgerechter Rechtsschutz bewirkt, dass sich das Recht in der Zeit verflüchtigt. Das Werk untersucht diese Abhängigkeit mit Blick auf das Verhältnis von Qualität und Schnelligkeit des Richterspruchs. Hierzu werden die verfassungs-, konventions- und unionsrechtlichen Grundlagen des Anspruchs auf richtiges Recht in angemessener Zeit herausgearbeitet, und es wird dargelegt, welche Rechtsfolgen sich bei einem Verstoß gegen diese Gewährleistungen ergeben. Rechtsvergleichend wird die Rechtslage in

\section{Die Kompensation der verlorenen Zeit - Wenn Prozesse Pause machen}

Das Gesetz über den Rechtsschutz bei überlangen Gerichtsverfahren und strafrechtlichen Ermittlungsverfahren Von VRiLSG Hermann Frehse

2017, ca. 1.325 S., brosch., ca. 198,- $-€$

ISBN 978-3-8487-3541-9

eISBN 978-3-8452-7874-2

(Bochumer Schriften zum Sozial-

und Gesundheitsrecht, Bd. 20)

Erscheint ca. Januar 2017

nomos-shop.de/28369

der Schweiz und in Österreich untersucht. Im zweiten Teil referiert das Werk unter kritischer Würdigung des Gesetzgebungsverfahrens die Genese des ÜGG. Im dritten Teil werden die $§ \S 198$, 200, 201 GVG eingehend analysiert. Dabei geht es nicht um die dogmatischen Grundlagen, sondern vorrangig um eine vertiefte Kommentierung der normativen Voraussetzungen für den Entschädigungsanspruch. Der vierte Teil gleicht ab, inwieweit die $\S \S 198$ ff. GVG den Vorgaben der EMRK entsprechen. 